\title{
Functional characterization of two defensin isoforms of the hard tick Ixodes ricinus
}

\author{
Tereza Chrudimská ${ }^{1,2^{*}}$, Jiřina Slaninová ${ }^{3}$, Nataliia Rudenko², Daniel Růžek ${ }^{1,2}$ and Libor Grubhoffer ${ }^{1,2}$
}

\begin{abstract}
Background: The immune system of ticks is stimulated to produce many pharmacologically active molecules during feeding and especially during pathogen invasion. The family of cationic peptides - defensins - represents a specific group of antimicrobial compounds with six conserved cysteine residues in a molecule.

Results: Two isoforms of the defensin gene (def1 and def2) were identified in the European tick Ixodes ricinus. Expression of both genes was induced in different tick organs by a blood feeding or pathogen injection. We have tested the ability of synthetic peptides def 1 and def 2 to inhibit the growth or directly kill several pathogens. The antimicrobial activities (expressed as minimal inhibition concentration and minimal bactericidal concentration values) against Gram positive bacteria were confirmed, while Gram negative bacteria, yeast, Tick Borne Encephalitis and West Nile Viruses were shown to be insensitive. In addition to antimicrobial activities, the hemolysis effect of def1 and def2 on human erythrocytes was also established.

Conclusions: Although there is nothing known about the realistic concentration of defensins in I. ricinus tick body, these results suggest that defensins play an important role in defence against different pathogens. Moreover this is a first report of a one amino acid substitution in a defensins molecule and its impact on antimicrobial activity.
\end{abstract}

\section{Background}

Ticks are known as vectors of severe human and animal diseases caused by viruses, bacteria and protozoa. Their immune system offers effective mechanisms against pathogenic microorganisms in the event of their permeation into the tick body. Some pathogen species (Babesia sp., Borrelia sp., Tick Borne Encephalitis Virus, etc.) can survive and colonize tick tissues and to be further transmitted to animal or human hosts during feeding [1].

The immune system of ticks and other arthropods possesses two sets of immune responses. The first one includes cellular responses represented by hemocytes and including encapsulation, nodulation and phagocytosis $[2,3]$. Hemocytes were shown to be one of the sites of immune molecule synthesis that play a role in humoral immune pathways [4]. The second set of immune responses in ticks consists of humoral responses, which involve the proteins that represent molecular factors of self/non-self recognition as well as effector molecules.

\footnotetext{
* Correspondence: terezam@paru.cas.cz

'University of South Bohemia, Faculty of Science, Branišovská 31, České

Buděovice, Czech Republic

Full list of author information is available at the end of the article
}

Defensins, naturally occurring antimicrobial peptides (AMPs), form the first line of defense against pathogens and have been found in a broad spectrum of living organisms: plants [5], mammals [6], insects [7-9], scorpions [10], mollusks [11] and several tick species [12-21]. Invertebrate defensins were first isolated from cultured cells of the flesh fly Sarcophaga peregrina [7]. Their amino acid sequence shows conservations across a broad phylogenetic range (ticks, insects, mollusks, and scorpions) suggesting these AMPs are relatively ancient effectors of innate immunity. Arthropod defensins are generally cationic AMPs having six cysteine residues forming disulfide bridges with the same pairing Cys1-Cys4, Cys2-Cys5, and Cys3-Cys6 [22]. In ticks, defensins are mainly expressed in the midgut after blood feeding or pathogen invasion $[14,23,24]$. Their antimicrobial activity is primarily directed against Gram-positive bacteria, but some isoforms are also effective against Gram-negative bacteria, yeasts and protozoa [24-27]. The initial interaction of defensins with bacterial cytoplasmic membranes involves electrostatic forces followed by permeabilization of the membranes and subsequently leading to bacterial lysis. It is thought that there may also be other, secondary, potential targets inside 
bacteria cells for cationic AMPs, such as nucleic acids or some enzymes [25,28].

Recently, multidrug resistant (MDR) bacterial strains have emerged and have rapidly spread in the environment, therefore the discovery of a novel class of antibiotics is urgently needed. During the course of evolution, nature has generated many molecules with conserved motifs that may represent specific probes for design of the new therapeutic agents. Characterization of these structures is of particular interest in order to produce molecules by chemical synthesis or recombinant systems, which may be candidates for new drug development [29]. Membraneactive cationic AMPs, like defensins, have the potential to become a new class of antibiotics with a new mode of interaction and promising therapeutical effects. These peptides are not yet affected by antibiotic-resistance mechanisms and, with various possible targets, the development of resistance might be difficult [28].

\section{Results}

\section{Antimicrobial and hemolytic activities of the synthetic} defensin isoforms

In the preliminary agar diffusion test, both synthetic isoforms of Ixodes ricinus defensin def1 and def2 revealed an antimicrobial activity against the Gram-positive bacteria Staphyloccocus xylosus, Micrococcus luteus, Bacillus subtilis and a clinical isolate of MDR strain of S. aureus (data not shown), but did not show any effect on Gram-negative bacteria (Escherichia coli, Pseudomonas aeruginosa) and yeast (Candida albicans). Subsequently the minimal inhibition concentrations (MICs) and the minimal microbicidal concentrations (MMCs) for selected sensitive bacteria species were determined. Synthetic def1 and def2 had an ability to inhibit Gram-positive bacteria in very low concentrations-specifically M. luteus (MIC $0.75 \mu \mathrm{M}$ and 0.37 $\mu \mathrm{M}$, respectively), B. subtilis (1.5 $\mu \mathrm{M}$ and $0.75 \mu \mathrm{M}$, respectively) and a methicilin resistant clinical isolate of S. aureus
- MRSA (50 $\mu \mathrm{M}$, and $25 \mu \mathrm{M}$, respectively). The minimal bactericidal concentrations were measured as the ability to kill $99.9 \%$ of bacteria in the culture. Both peptides showed the ability to kill tested bacteria within one hour, thought the MMCs of both peptides were slightly higher than the MICs. The values are summarized in Table 1 .

Def1 as well as def2 isofoms exhibited no virucidal effect on the Tick Borne Encephalitis virus (TBEV), and West Nile virus (WNV). Moreover, no significant inhibition of TBEV, and WNV growth in a culture of PS cells was seen in the presence of the compounds. The only decrease (approx. $1 \log _{10} \mathrm{pfu} / \mathrm{ml}$ ) in WNV titer was seen in PS cell culture at 24 hours post-infection (p.i.) in the presence of def2 when compared to untreated control. However, no difference in virus titer between cultures treated with def2 and untreated controls was seen at 48 hours p.i. (data not shown). In conclusion, I. ricinus defensin isoforms did not reveal any substantial antiviral activity against TBEV, as a representative of tick-transmitted viral pathogens, and WNV, as a representative of mosquito-borne viruses.

To determine the effects of I. ricinus defensins on mammalian cells, we measured the level of hemolysis caused by def1 and def2 in concentrations effective at killing Gram-positive bacteria. The results show that both peptides are harmless to human erythrocytes in concentrations of up to $12.5 \mu \mathrm{M}$ ( $\mathrm{p}<0.05)$. In these concentrations def1 and def2 caused $2.9 \%$ and $2.0 \%$ hemolysis, respectively. The hemolysis rose to $75 \%$ and $64.6 \%$ respectively, when the highest concentrations of def1 and def2 were used (Figure 1).

The amino acid sequences of def1 and def2 differ only in one amino acid residue in position 8 , where phenylalanine exists in case of def1 and arginine in case of def2. This substitution results in slightly different antimicrobial potential of def1 and def2. Def2 isoform is more effective in the inhibition of bacteria cell growth and killing of bacteria than def1 peptide. However, no

Table 1 Minimal inhibition concentrations (MICs) and minimal microbicidal concentrations (MMCs) of def1 and def2 isoforms

\begin{tabular}{|c|c|c|c|c|}
\hline \multirow[t]{2}{*}{ Bacterial, yeast and viral species } & \multicolumn{2}{|c|}{$\mathrm{MIC}[\mu \mathrm{M}]$} & \multicolumn{2}{|c|}{ MMC $[\mu \mathrm{M}]$} \\
\hline & def1 & def2 & def1 & def2 \\
\hline Bacillus subtilis (G+) & 1.5 & 0.75 & 2 & 1 \\
\hline Micrococcus luteus (G+) & 0.75 & 0.37 & 10 & 5 \\
\hline Staphylococcus luteus (G+) & 50 & 25 & 50 & 25 \\
\hline Escherichia coli (G-) & no effect ${ }^{a}$ & no effect $^{a}$ & & \\
\hline Pseudomonas aeruginosa (G-) & no effect ${ }^{b}$ & no effect ${ }^{b}$ & & \\
\hline Candida albicans (yeast) & no effect $^{b}$ & no effect ${ }^{b}$ & & \\
\hline TBEV (Flaviviridae) & no effect ${ }^{b}$ & no effect ${ }^{b}$ & no effect $^{b}$ & no effect ${ }^{b}$ \\
\hline WNV (Flaviviridae) & no effect ${ }^{b}$ & no effect ${ }^{b}$ & no effect ${ }^{b}$ & no effect ${ }^{b}$ \\
\hline
\end{tabular}

Determination of MICs and MMCs for each peptide and bacteria was performed at least 3-times in doublets. Def2 peptide was significantly more efficient in the inhibition of growing G-positive bacteria $(\mathrm{G}+)$ in cultures and in microbicidal experiments. Gram negative bacteria (G-), as well as yeast, WNV and two strains of TBEV (Hypr and Neudoerfl), were not influenced by these peptides ( ${ }^{a}$ no effect up to $50 \mu \mathrm{M} ;{ }^{b}$ no effect up to $\left.100 \mu \mathrm{M}\right)$. 




Figure 1 Hemolytic effect of def1 and def2. Hemolytic activity was carried out using $2 \%$ (vol/vol) erythrocyte suspension to evaluate all mild impacts on the erythrocyte membrane [40]. Normally, the $4-5 \%$ ( $\mathrm{vol} / \mathrm{vol}$ ) erythrocyte suspension is used for the hemolysis experiments $[41,42]$. Significant differences between negative controls and peptides are marked by an asterisk $(p<0.05)$. No differences between hemolysis by def1 and def2 were statistically determined.

significant difference was noticed in the degree of erythrocyte hemolysis.

Stage- and tissue-specific expression profiles of I. ricinus defensin isoforms

Semi-quantitative two step RT-PCR was performed to determine defensin gene expression in all developmental stages and in selected tissues before and after blood feeding or hemocel injection of different substances. Expression of def1 mRNA was induced by blood intake at all life stages - larva, nymph and imago, while def 2 mRNA expression was detected only in adult females with a significant increase after a blood meal (Figure 2). Def1 was expressed in mitgut in both cases-before and after blood meal, while its expression in Malphigian tubes and ovaries was induce after blood intake or injection of different compounds into blood fed female, respectively (see Figure 2 , 3a). Results of tissue specific expression patterns analysis for $I$. ricinus def 2 isoform in response to blood feeding, injection of bacteria or sterile water (Figure 2, 3a,) showed that expression of def 2 isoform was present in midgut and ovaries of unfed female after injection and blood feeding was a big stimulus for def 2 expression in all tested organs (Figure 2, 3b) House-keeping gene, $\beta$-actin, was expressed at the same expression levels in all samples (Figure 2, 3c).

Newly designed primers for amplification of $I$. ricinus $\beta$-actin gene were confirmed as specific not only for $I$. ricinus, but also for the I. scapularis and Dermacentor marginatus $\beta$-actin gene. The obtained products were sequenced and partial sequences of $\beta$-actin gene of $I$. ricinus and $D$. marginatus were submitted to GeneBank (accession no. HQ682101; HQ645110, respectively).

\section{Discussion}

\section{Functional characterization of def1 and def2}

Molecules involved in the mechanism of innate immunity of invertebrates are a rich source of potential novel candidates for antibiotic development. Antimicrobial proteins (AMPs) - mainly defensins, play an important role in invertebrate immunity pathways. Recently there has been greater attention paid to this protein family. Here, we present the functional characterization of Ixodes ricinus defensins (def1, def2), previously described in our lab $[18,19]$. Defensins are known to be effective mainly against Gram-positive bacteria [24,25,27]. Our results confirmed this statement showing that $I$. ricinus defensin isoforms possess an effective antimicrobial activity against Gram positive bacteria S. xylosus, M. luteus, B. subtilis and MRSA clinical isolate $S$. aureus. Def1 and def2 isoforms show a strong ability to inhibit Gram-positive bacteria growth in low doses (see Table 1). Furthermore, def1 and def 2 antimicrobials have the advantage of being bactericidal. Within one hour, $99.9 \%$ of tested bacteria were killed and observed MMCs corresponded to MICs, showing






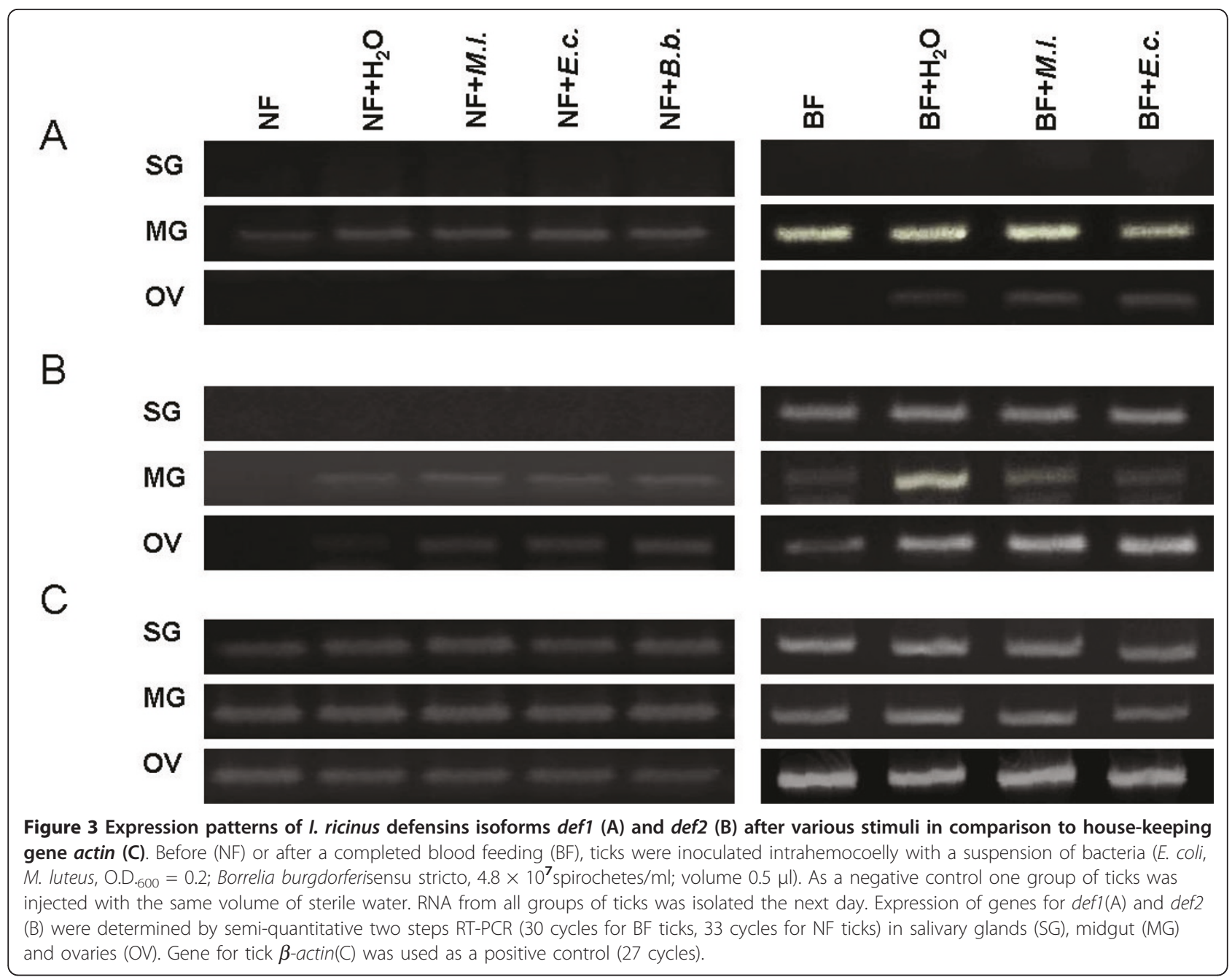

high efficiency of I. ricinus defensins. Neither def1 nor def 2 affected the growth of tested Gram-negative bacteria (E. coli and a multi-resistant clinical isolate of $P$. aeruginosa) and yeast $C$. albicans in tested concentrations (Tab. 1). Generally, Gram-negative bacteria are more resistant to treatment of cationic antimicrobial peptides. Their cell wall, mainly the outer membrane, is known to be an effective permeability barrier $[27,30]$. It is possible that this is the reason the peptides cannot directly interact with the cell membrane. Despite the fact that the majority of findings showed tick defensins as mainly anti Gram-positive bacteria peptides, longicin, the defensin of the Asian tick Haemaphysalis longicornis, was also able to inhibit Gramnegative bacteria (P. aeruginosa, E. coli), a fungus (Pichia pastoris) and merozoites of an intracellular parasite Babesia equi [26]. Weak antibacterial activity of I. persulcatus defensin was observed against the Gram-negative bacterium E. coli [24].

Bacteria naturally co-existing with the tick, such as the symbiotic bacterium Stenotrophomonas maltophila (from the midgut of the tick I. persulcatus), the pathogenic spirochete Borrelia garinii (Ixodes ticks) or I. persulactus-derived Bacillus sp., revealed the natural resistance to tick defensins [27,31]. Here we have demonstrated that TBEV (virus derived from I. ricinus tick) and its life cycle were not influenced by tick defensins def1 and def2. Lyme disease spirochetes (B. garinii), classified as Gram-negative bacteria, were not sensitive to defensins synthesized according to the sequences derived from vector (Ixodes ricinus, def1) and non-vector ticks Haemaphysalis longicornis or Ornithodoros moubata (defC). [27].

Although the antiviral activity of several defensins (human, murine) was reported, the I. ricinus defensins did not substantively influence WNV and its life cycle in addition to TBEV [32,33].

Therapeutic alternatives for the treatment of multidrug resistant bacteria infections are restricted to antibiotics introduced recently to clinical practice [34]. Recently, many human pathogens have emerged with 
multi resistance to known antibiotics [35]. Therefore, the basic antimicrobial testing should be a part of all research with probable or expected antimicrobial molecules. As was mentioned earlier, I. ricinus def1 and def2 showed antibacterial activity against G-positive bacteria. A methicilin resistant clinical isolate of $S$. aureus (MRSA) was sensitive to these peptides as well. Even if the MIC and the MMCs values of def1 and def2 peptides against $S$. aureus (MRSA) were higher than in other tested Gram-positive bacteria, this finding showed the ability of defensins to inhibit and kill pathogenic bacterium with multi-drug resistance. In contrast, $S$. aureus strain Wood was more sensitive $\left(\mathrm{MIC}_{\mathrm{def} 1} 40\right.$ $\mu \mathrm{g} / \mathrm{ml}$; MW 4231.9; equivalent $9.45 \mu \mathrm{M}$ concentration) than the MRSA strain to Ixodes ricinus def1 peptide treatment [27].

These findings, supplemented by the fact that these defensins have relative low toxicity to human cells (measured by the degree of red cell hemolysis; Figure 1), suggest that $I$. ricinus tick defensins (mainly def2 isoform) are possible candidates for new antimicrobial (especially anti-MRSA species) drug development with potential topical application.

\section{Impact of defensin primary structure on its function}

The importance of forming the intra-molecular disulphide bonds between six cysteine residues in the defensin molecule had been reported before and was summarized by Ganz and Lehrer [22]. Specific S-S formation (between Cys1 - Cys4, Cys2 - Cys5 and Cys3 - Cys6) is vital for defensin function. The linear synthetic defensin from I. persulcatus displayed weak antibacterial activity in the growth inhibition test in comparison to the folded peptide with a confirmed tertiary structure of the same defensin peptide [31]. Nevertheless, Tsuji and colleagues [26] showed that the most important part of defensin molecule responsible for antimicrobial activity is the sequence of the C-terminal 20 amino acids. This shorter peptide (signed as $\mathrm{C} 4$ peptide) exerts the same antibacterial activity as a full length and folded recombinant mature defensin.

Here we have shown that a single amino acid substitution on the N-terminal of defensin molecule also influences the function of the peptide. Def1 and def2 isoforms differ in position 8 of the amino acid sequences. The substitution affects the ability to kill previously mentioned bacteria: def2 (Arg8) possesses greater ability to inhibit bacterial growth and kill bacteria than def1 isoform (Phe8). While the substitution doesn't affect the predicted tertiary structure of both peptides (Figure 4a, b), the peptide surface has changed: the amino acid residues of Phe8 and Arg8 are present on the surface (see Figure $4 c, d)$. The two peptides also differ in cationicity - def 2 has higher predicted positive charge (7.052; at $\mathrm{pH} 7.0)$ and could probably be more efficient in bacteria cell membrane depolarization than isoform def1 (6.052; at $\mathrm{pH}$ 7.0).

\section{Defensins expression patterns}

During feeding, ticks are naturally exposed to host blood environment possibly containing infectious agents. Activation of the tick innate immune responses is necessary for a successful defense against invading pathogens, and consequently for completing blood intake and the life cycle. Defensins, as a part of humoral immunity pathway, are induced by many stimuli. They are mainly up-regulated in the midgut by blood feeding $[13,14,24,26]$. It is speculative if defensins may play a role in blood digestion - our results show that def1 as well as def 2 are able to slightly lyse erythrocytes up to $12.5 \mu \mathrm{M}$ concentration. In our opinion a more probable explanation for the up-regulation of defensin gene expression after blood intake is the activation of the immune system due to history events of simultaneously intake of pathogens and blood meal. The results of gene expressions show defensins as the first line defense molecules in tick immunity. Gene for def1 was predominantly expressed in the midgut, while def2 was detected in all tested organs after different treatments. Our observations reveal that the immune system of I. ricinus females (measured by defensin expression) reacts on different pathogens almost equally. Analogously, there were reports of up-regulation of defensins gene expressions after a bacterial treatment in several other ticks. In the soft tick, Ornithodoros moubata, four defensin isoforms were induced by injection of different bacteria or bacteria cell wall components. These O. moubata defensin isoforms react slightly different to treatment of Gram positive or Gram negative bacteria [25]. Infection of the hard tick $D$. variabilis by Gram-negative bacteria Anaplasma marginale also leads to increasing defensin gene mRNA levels [23].

\section{Conclusions}

In conclusion, I. ricinus defensins (def1, def2) play an important role in the tick's innate immunity. Their expression is mainly induced by a blood feeding - the most important process for pathogen entry. Therefore, the expression of both defensin isoforms is predominantly found in the midgut but sometimes in other organs such as salivary glands or ovaries as well. Both I. ricinus defensin isoforms possess bactericidal properties with potency predominantly against Gram-positive bacteria; the def2 isoform was found to be more effective in killing bacteria with the same level of human erythrocyte hemolysis as the def1 isoform. Generally, with the increasing emergence of multi-resistant bacteria, tick defensins as well as other 
A

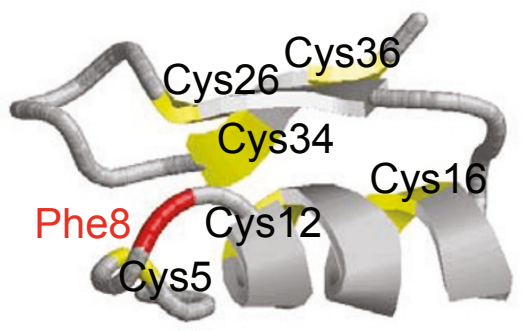

C

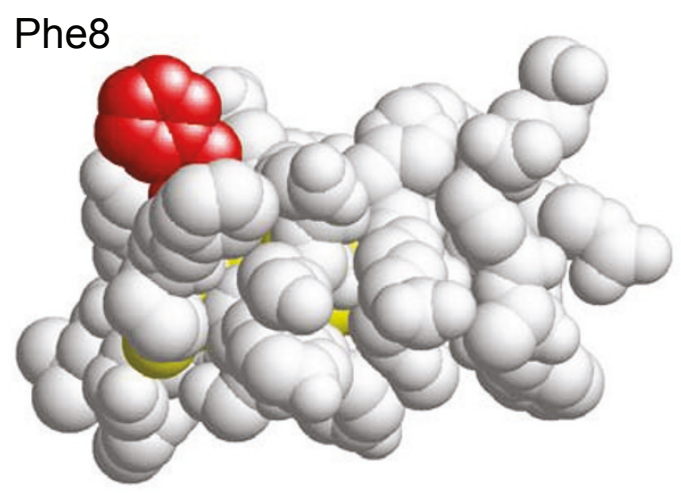

B

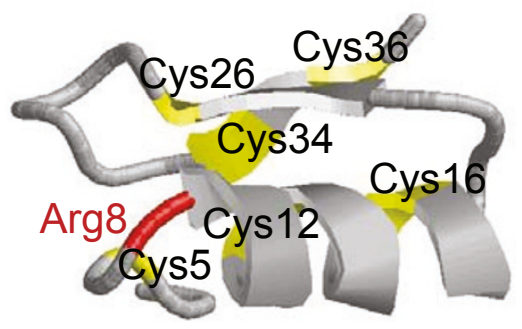

D

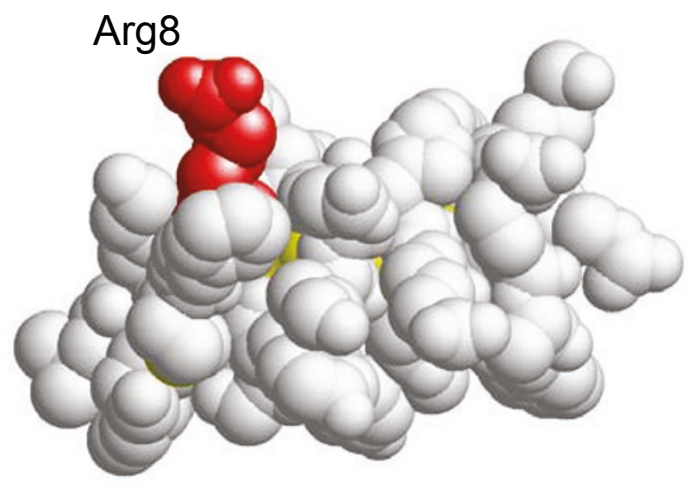

Figure 4 Predicted 3D structures of def1 (A) and def2 (B) peptides and the impact of amino acid substitution on molecular surface (C, D). The predicted 3D structures (A, B) show no significant differences, but the amino acids residues of Phe8 (def1) and Arg8 (def2) projected out of the molecule surface (C, D; respectively). Because the arginine carries out the positive charge, the impact on molecule def2 cationicity is without doubt. Seeing that the defensins mode of action is a thorough depolarization of the bacteria cytoplasm membrane [25], the cationicity of peptides could be the explanation for def2 higher efficiency in killing bacteria. The predicted tertiary structures of def1 and def2 peptides were based on the template 1 fjnA - 3D structure of defensin MGD-1 from the mollusk Mytilus galloprovincialis[43]. Sequence similarity between MGD-1 and def1, def2 were 48.65\% in both cases.

arthropod defensins can be expected to be introduced to the medical field as new molecules with antibacterial activity.

\section{Methods}

\section{Tick samples}

I. ricinus ticks were provided by the Biological Centre, Institute of Parasitology, Academy of Science of the Czech Republic. Uninfected ticks of different life stages (larva, nymph and imago) were fed on adult guinea pigs (infection-free animals treated in accordance with the Animal Protection Law of the Czech Republic no. 246/ $1992 \mathrm{Sb})$. Ticks were collected after completing blood feeding (self-separation from the animal).

\section{Semi-quantitative two step RT-PCR analysis}

Semi-quantitative two-step reverse transcriptase-polymerase chain reaction (RT-PCR) analysis was performed to analyze the gene expression of Ixodes ricinus defensins. Total RNA was extracted from all tick samples using the TRI reagent (Sigma, USA) according to the manufacturers' recommendations. RNA samples were obtained from unfed and fed larvae, nymphs and adult females and from dissected tissues unfed and fed females (hemolymph, midgut, salivary glands, ovary, and Malphigian tubes).

Single strand cDNA was prepared from the total RNA with random primers $(0.2 \mu \mathrm{g}$ per reaction) using RevertAid H Minus First Strand cDNA Synthesis Kit (MBI Fermentas, Lithuenia). Five micrograms of total RNA was used per each reaction. Synthesis of the first strand cDNA was carried out at $42^{\circ} \mathrm{C}$ for 60 minutes according to the manufacturers' recommendations.

Primers were designed according to I. ricinus defensin isoforms sequences previously obtained in our laboratory $[18,19]$. The mature defensins isoforms (def 1, def 2) were amplified using specific primers: Def1ma forward 5'GGT GGC TAC TAC TGC CCA TTT TTT-3', Def2ma forward 5'- GGT GGT TAC TAC TGC CCA TTC CG3'. The reverse primer (DefRma) sequence (5'- TCA GAC 
GCA GAT GCA GGT CTT TT-3') was the same for both defensin isoforms. The tick housekeeping gene encoding $\beta$-actin was used as a control in RT-PCR using the gen-specific primers; the primers were designed according to Dermacentor variabilis (EF488512) and Rhipicephalus (Boophilus) microplus (AY255624) actin sequences and were as follows (forward primer 5'-ATG TGT GAC GAC GAG GTT GCC GC-3', reverse primer 5'-GTA CAG CGA CAG CAC GGC CTG G-3'). To amplify the target gene, the single strand cDNA (150 ng per reaction) was used. Amplification was performed with GoTaq Colorless Master Mix (Promega, USA) in 20 $\mu l$. PCR conditions were as follows: $5 \mathrm{~min}$ at $96^{\circ} \mathrm{C}$, followed by 28 cycles at $96^{\circ} \mathrm{C}$ for $25 \mathrm{~s}, 53^{\circ} \mathrm{C}$ (defensins) or $50^{\circ} \mathrm{C}$ (actin) for $30 \mathrm{~s}$ and $72^{\circ} \mathrm{C}$ for $1 \mathrm{~min}$. Final extension step was at $72^{\circ} \mathrm{C}$ for $5 \mathrm{~min}$. PCR was performed in a Mastercycler (Eppendorf, Germany) thermal cycler. Products of the reaction were separated by electrophoresis on $1.8 \%$ agarose gel electrophoresis.

Def1 and def2 PCR products, as well as the $\beta$-actin gene PCR product, were cut off the gel and purified using the QIAquick Gel Extraction Kit (Qiagen, USA) and subsequently cloned into $\mathrm{pCR}^{\circledR} 4$-TOPO vector (Invitrogen, USA) for sequencing to proof defensin isoform primers specificity. DNA sequencing was performed using ABI 3130 Sequencer and the Big Dye ${ }^{\circledR}$ Terminator v3.1 Cycle Sequencing Kit (Applied Biosystems, USA) with M13 forward/reverse primers. Sequences were analyzed with DNASTAR software (DNASTAR, United Kingdom). BLAST programs of the National Centre for Biotechnology Information (Bethesda, USA) were used for similarity search using the data available in GenBank ${ }^{\circledR}$.

\section{Preparation of synthetic tick defensins}

Peptides def1 and def2 (mature parts, both 37 amino acid long) were custom synthesized according to putative amino acid sequences (accession numbers AAP94724 and ABC88432, respectively) by the Peptide 2.0 Company (Chantilly, VA, USA). Purification by reversed-phase highperformance liquid chromatography and MS analyses were performed by the vendor. According to the data reports supplied with the products, synthetic Def1 (GGYYCPFFQDKCHRHCRSFGRKAGYCGGFLKKTCICV) was $93.84 \%$ pure and its molecular weight was $4.237 \mathrm{kDa}$, while synthetic Def2 (GGYYCPFRQDK CHRHCRSFGRKAGYCGGFLKKTCICV) was $\mathbf{8 5 . 8 2 \%}$ pure and its molecular weight was $4.247 \mathrm{kDa}$. The products were supplied as lyophilized trifluoroacetate salt which was stored at $-76^{\circ} \mathrm{C}$. Before experiments, the peptides were dissolved in PBS buffer containing 0.05\% Tween 20 (Loba Feinchemie, Austria) and $1 \mu \mathrm{M} \beta$-merkaptoethanol (Bio-Rad, CA, USA) to final $1 \mathrm{mM}$ concentration.

\section{Biological activity assays}

Following organisms were used for determination of basic antimicrobial activity profile: Bacillus subtilis (B. s.) 168, kindly provided by Prof. Yoshikawa (Princeton University, Princeton, NJ, USA); Escherichia coli (E. c.) B No. CCM 7372 and Micrococcus luteus (M. l.) No. CCM 144 from the Czech Collection of Microorganisms, Brno; Staphylococcus aureus (S. a.) and Pseudomonas aeruginosa (P. a.) were obtained as multi-resistant clinical isolates, No. 4231 and 8567, respectively, from Liberec Hospital, Czech Republic; and Borrelia burgdorferi (B.b.) No. ATCC 35211 from American Type Culture Collection, Manassas, VA, USA. Yeast, Candida albicans F7-39/ IDE99, kindly provided by the Institute of Organic Chemistry and Biochemistry, was a clinical strain from a collection of fungi at the Institute of Microbiology, Faculty of Medicine and Dentistry, Palacký University Olomouc, Czech Republic. For antiviral activity assay, two representatives of TBEV (strains Neudoerfl, and Hypr) and one isolate of WNV were used. The strain Neudoerfl was originally isolated from $I$. ricinus tick in Austria in 1971 [36], and the strain Hypr was isolated from the blood of a 10-year-old child with tick-borne encephalitis in 1953 [37]. Low-passage TBEV strains were used in this study. WNV isolate used in this study is of unknown origin and underwent 28 passages in brains of suckling mice.

\section{Antimicrobial assay}

Qualitative estimates of the antimicrobial activities of both synthesized I. ricinus defensin isoforms were performed by the agar dilution double-layer technique. One hundred micro litters of fresh bacterial culture (approximately $10^{7}$ colony forming units per Petri dish) prepared in LB broth were added to $2 \mathrm{ml}$ of melted soft agar $(0.5 \%$ LB agar) once temperature decreased to approximately $42^{\circ} \mathrm{C}$. Subsequently, soft agar was poured over the surface of the Petri dish containing $20 \mathrm{ml}$ of $2 \%$ LB agar. Synthetic defensins 1 and 2 were diluted to concentration of $100 \mu \mathrm{M}$ in physiological buffer and one microliter of either peptide was dropped on the surface of the solidified upper layer containing bacteria. As a negative control, dissolving buffer (PBS containing 0.05\% Tween 20 and $1 \mu \mathrm{M} \beta$-merkaptoethanol) was used. Plates were incubated at $37^{\circ} \mathrm{C}$ overnight.

\section{Minimal inhibition concentratins (MICs)}

Quantitatively, MICs were established by observing bacteria growth in multi-well plates. Stock solutions (1 mM) of def1 and def 2 were diluted in LB broth and put into the wells of multi well plates. Bacteria in mid-exponential phase were diluted $\left(1 \times 10^{5} \mathrm{CFU} / \mathrm{ml}\right)$ and added to the wells with peptides $(0.1 \mathrm{ml}$ of diluted peptide and $0.1 \mathrm{ml}$ of bacteria culture), final peptide concentration ranged from 0 to $100 \mu \mathrm{M}$. Diluted dissolving buffer in LB broth 
was used as a negative control. Multi-well plates were incubated in a Bioscreen $\mathrm{C}$ instrument (Helsinki, Finland) for $20 \mathrm{~h}$ (or $24 \mathrm{~h}$ for $M$. luteus) at $37^{\circ} \mathrm{C}$, with constant shaking. The absorbance was measured at $540 \mathrm{~nm}$ every $15 \mathrm{~min}$, and each peptide was tested in 3 independent experiments.

\section{Minimal micorbicidal concentrations (MMCs)}

The overnight bacterial cultures of M. luteus, B. subtilis and S. aureus were subcultured in fresh $\mathrm{LB}$ media at $37^{\circ} \mathrm{C}$ with shaking to obtain log-phase bacterial cells. Subsequently, bacteria cells were diluted to $1-2 \times 10^{6}$ cells $/ \mathrm{ml}$ in PBS (pH 7.4). One hundred microlitres of cell suspension were mixed with $100 \mu \mathrm{l}$ of peptide def1 or def 2 solutions $(0-200 \mu \mathrm{M})$ and incubated 1 hour without shaking at $37^{\circ} \mathrm{C}$. Afterward the bacteria samples were diluted either 100 or 1000 times, spread on LB agar plates and incubated overnight at $37^{\circ} \mathrm{C}$. Grown colonies were counted, CFU per $\mathrm{ml}$ and percentage of dead bacteria relative to controls was calculated. MMCs were evaluated as the lowest peptide concentration able to kill $99.9 \%$ of bacteria in 3 independent experiments.

\section{Antiviral activity}

Virucidal effect of defensin 1 and 2 on TBEV was investigated by incubation of $10^{5} \mathrm{pfu}$ of the virus in L15 medium (3\% FCS) in the presence or absence of the def1 and def 2 compounds at final concentration of $100 \mu \mathrm{M}$ for 1 hour at $37^{\circ} \mathrm{C}$. The residual infectivity was determined by a plaque assay as described previously [38].

The inhibitory effect of def1 and def2 on TBEV replication was investigated on PS cell monolayers (porcine kidney stable) [39]. The cells in 96-well plates were infected with $10^{5}$ pfu per well in L15 medium (3\% FCS). After one hour of adsorption at $37^{\circ} \mathrm{C}$, the inoculum was removed by washing the cells with PBS. Subsequently, the cells were incubated in L15 medium in the presence or absence of the peptides. The concentration of defensins used in the experiment was $100 \mu \mathrm{M}$. Supernatant media from the wells were collected at 24 and 48 hours postinfection and stored at $-70^{\circ} \mathrm{C}$ until processing. Virus titer was determined by the plaque assay as described previously [38].

\section{Hemolytic assay}

EDTA-anticoagulated human venous blood was obtained from 2 young healthy volunteers. Erythrocytes were harvested by centrifugation $\left(2000 \mathrm{rpm}, 10 \mathrm{~min}\right.$, and $20^{\circ} \mathrm{C}$ ) and washed three times with sterile PBS. Suspension of erythrocytes $(2 \%$; vol/vol) was used for the assay. Stock solution of peptides def 1 and 2 were diluted in PBS and co-incubated with erythrocytes $\left(37^{\circ} \mathrm{C}, 2\right.$ hours $)$ in the final volume of $200 \mu \mathrm{l}$ and final concentrations from 0.37 $\mu \mathrm{M}$ to $100 \mu \mathrm{M}$. After incubation, the suspension was centrifuged $\left(2000 \mathrm{rpm}, 10 \mathrm{~min}\right.$, and $20^{\circ} \mathrm{C}$ ), $100 \mu \mathrm{l}$ of supernatant was removed, and the absorbance of samples was measured at $405 \mathrm{~nm}\left(\mathrm{~A}_{\mathrm{N}}\right)$. The hemolytic activity was calculated in correlation to negative and positive controls $\left(\%\right.$ hemolysis $=\left(\mathrm{A}_{\mathrm{N}}-\mathrm{A}_{0} / \mathrm{A}_{100}-\mathrm{A}_{0}\right) \times 100 ; \mathrm{A}_{0}=0 \%$ hemolysis in PBS; $\mathrm{A}_{100}=100 \%$ hemolysis obtained by incubation with $0.2 \%$ solution of Triton X-100 in PBS).

\section{Statistical analysis}

The significance of any differences obtained between def 1 and def 2 peptides, concentrations used and controls was evaluated by the Mann-Whitney U test, nonparametrical (Statistica 6.0, Statsoft, Inc. Tulsa, USA). The results represent 5 similar doublet experiments for hemolytic activity and 3 doublet tests for antimicrobial activity.

\section{Tertiary structure prediction}

For prediction of the 3-D structure of both def1 and def2 peptides, the Swiss-Model Protein Modeling Server was used [http://swissmodel.expasy.org/SWISS-MODEL. html] to find the homologues for I. ricinus molecules. A pdb file of the molecule (according to the similar sequences of structures from the protein databank) was downloaded and used in RasWin Molecular Graphics program (Version 2.7.5; http://www.rasmol.org/) to view the 3D structure of both peptides.

\section{Acknowledgements}

We are grateful to Jan Erhart for his help during work with the ticks and to Tomáš Chrudimský for his help during modeling of 3-D structures of both peptides.

This work was partially supported by the grants MSM 6007665801 and LC06009 from Ministry of Education, Youth and Sports of the Czech Republic, institutional research grant Z60220518 (Academy of Sciences of the (zech Republic), as well as grants 206/09/1782, 206/09/H026 and P302/ 11/1901 of the Czech Science Foundation.

\section{Author details}

'University of South Bohemia, Faculty of Science, Branišovská 31, České Buděovice, Czech Republic. ${ }^{2}$ Biology Centre v.v.i., ASCR, Institute of Parasitology, Branišovská 31, České Buděovice, Czech Republic. Institute of Organic Chemistry and Biochemistry, ASCR, Flemingovo square 2, Prague, Czech Republic.

\section{Authors' contributions}

TC carried out molecular biology work, functional analysis, and statistics; participated in MIC establishment and antiviral tests and drafted the manuscript. JS carried out MIC establishment. DR carried out the antiviral activity tests. LG and NR participated in the study design and helped to draft the manuscript. All authors read and approved the final manuscript.

\section{Competing interests}

The authors declare that they have no competing interests.

Received: 16 February 2011 Accepted: 19 April 2011

Published: 19 April 2011

\section{References}

1. Nuttall PA, Labuda M: Saliva-assisted transmission of tick-borne pathogens. In Ticks - Biology, Diseases and Control. Edited by: Bowman AS, Nuttall PA. Cambridge University Pres; 2008:205-219.

2. Eggenberger $L R$, Lamoreaux WJ, Coons LB: Hemocytic encapsulation of implants in the tick Dermacentor variabilis. Exp Appl Acarol 1990, 9:279-287. 
3. Gillespie JP, Kanost MR, Trenczek T: Biological mediators of insect immunity. Ann Rev Entomol 1997, 42:611-643.

4. Taylor DM: Innate Immunity in Ticks: A review. J Acarol Soc Jpn 2006, 15:109-127.

5. Broekaert WF, Terras FR, Cammue BP, Osborn RW: Plant defensins: novel antimicrobial peptides as components of the host defense system. Plant Physiol 1995, 108:1353-1358.

6. Selsted ME, Harwig SS, Ganz T, Schilling JW, Lehrer Rl: Primary structures of three human neutrophil defensins. J Clin Invest 1985, 76:1436-1439.

7. Matsuyama K, Natori S: Purification of three antibacterial proteins from the culture medium of $\mathrm{NIH}-\mathrm{Sape}-4$, an embryonic cell line of Sarcophaga peregrina. J Biol Chem 1988, 263:17112-17116.

8. Lowenberger C, Bulet P, Charlet M, Hetru C, Hodgeman B, Christensen BM, Hoffmann JA: Insect immunity: isolation of three novel inducible antibacterial defensins from the vector mosquito, Aedes aegypti. Insect Biochem Mol Biol 1995, 25:867-873.

9. Viljakainen L, Pamilo P: Identification and molecular characterization of defensin gene from the ant Formica aquilonia. Insect Mol Biol 2005, 14:335-338.

10. Cociancich S, Goyffon M, Bontems F, Bulet P, Bouet F, Menez A Hoffmann JA: Purification and characterization of a scorpion defensin, a $4 \mathrm{kDa}$ antimicrobial peptide presenting structural similarities with insect defensins and scorpion toxins. Biochem Biophys Pes Commun 1993, 194:17-22

11. Charlet M, Chernysh S, Philippe H, Hetru C, Hoffmann JA, Bulet P: Innate immunity: Isolation of several cysteine-rich antimicrobial peptides from the blood of a mollusc, Mytilus edulis. J Bioch Chem 1996, 271:21808-21813.

12. Johns R, Sonenshine DE, Hynes WL: Identification of a defensin from the hemolymph of the American dog tick, Dermacentor variabilis. Insect Biochem Mol Biol 2001, 31:857-865.

13. Nakajima $Y$, van der Goes van Naters-Yasui A, Taylor D, Yamakawa M: Two isoforms of a member of the arthropod defensin family from the soft tick, Ornithodoros moubata (Acari: Argasidae). Insect Biochem Mol Biol 2001, 31:747-751.

14. Nakajima Y, van der Goes van Naters-Yasui A, Taylor D, Yamakawa M: Antibacterial peptide defensin is involved in midgut immunity of the soft tick, Ornithodoros moubata. Insect Mol Biol 2002, 11:611-618.

15. Ceraul SM, Sonenshine DE, Ratzlaff RE, Hynes WL: An arthropod defensin expressed by the hemocytes of the American dog tick, Dermacentor variabilis (Acari: Ixodidae). Insect Biochem Mol Biol 2003, 33:1099-1103.

16. Fogaça AC, Lorenzini DM, Kaku LM, Esteves E, Bulet P, Daffre S: Cysteinerich antimicrobial peptides of the cattle tick Boophilus microplus: isolation, structural characterization and tissue expression profile. Dev Comp Immunol 2004, 28:191-200.

17. Hynes WL, Ceraul SM, Todd SM, Seguin KC, Sonenshine DE: A defensin-like gene expressed in the black-legged tick, Ixodes scapularis. Med Vet Entomol 2005, 19:339-344.

18. Rudenko N, Golovchenko M, Edwards MJ, Grubhoffer L: Differential expression of Ixodes ricinus tick genes induced by blood feeding or Borrelia burgdorferi infection. J Med Entomol 2005, 42:36-41.

19. Rudenko N, Golovchenko M, Grubhoffer L: Gene organization of novel defensin of Ixodes ricinus: first annotation of an intron/exon structure in a hard tick defensin gene and first evidence of the occurrence of two isoforms of one member of the arthropod defensin family. Insect Mol Biol 2007, 16:501-507.

20. Chrudimská T, Chrudimský T, Golovchenko M, Rudenko N, Grubhoffer L: New defensins from hard and soft ticks: Similarities, differences, and phylogenetic analyses. Vet Parasitol 2010, 167:298-303.

21. Jaworski DC, Zou Z, Bowen CJ, Wasala NB, Madden R, Wang Y, Kocan KM, Jiang $\mathrm{H}$, Dillwith JW: Pyrosequencing and characterization of immune response genes from the American dog tick, Dermacentor variabilis (L.). Insect Mol Biol 2010, 19:617-30.

22. Ganz T, Lehrer Rl: Defensins. Curr Opin Immunol 1994, 6:584-589.

23. Kocan KM, de la Fuente J, Manzano-Roman R, Naranjo V, Hynes WL, Sonenshine DE: Silencing expression of the defensin, varisin, in male Dermacentor variabilis by RNA interference results in reduced Anaplasma marginale infections. Exp Appl Acarol 2008, 46:17-28.

24. Saito $Y$, Konnai S, Yamada S, Imamura S, Nishikado H, Ito T, Onuma M, Ohashi K: Identification and characterization of antimicrobial peptide, defensin, in the taiga tick, Ixodes persulcatus. Insect Mol Biol 2009, 18:531-539.

25. Nakajima Y, Ishibashi J, Yukuhiro F, Asaoka A, Taylor DM, Yamakawa M: Antibacterial activity and mechanism of action of the tick defensin against Gram-positive bacteria. Biochim Biophys Acta 2003, 1624:125-130.

26. Tsuji N, Battsetseg B, Boldbaatar D, Miyoshi T, Xuan X, Oliver JH Jr, Fujisaki K: Babesial vector tick defensin against Babesia sp. parasites. Infect Immun 2007, 75:3633-3640.

27. Isogai $\mathrm{E}$, Isogai $\mathrm{H}$, Takahashi K, Kobayashi-Sakamoto M, Okumura K: Antimicrobial activity of three tick defensins and four mammalian cathelicidin-derived synthetic peptides against Lyme disease spirochetes and bacteria isolated from the midgut. Exp Appl Acarol 2009, 49:221-228.

28. Hancock REW: Cationic peptides: effectors in innate immunity and novel antimicrobials. Lancet Infect Dis 2001, 1:156-164.

29. Bulet $P$, Stöcklin R, Menin L: Anti-microbial peptides: from invertebrates to vertebrates. Immunol Rev 2004, 198:169-184.

30. Nikaido $\mathrm{H}$ : Preventing drug access to targets: cell surface permeability barriers and active efflux in bacteria. Semin Cell Dev Biol 2001, 12:215-223.

31. Isogai E, Isogai H, Okumura K, Hori H, Tsuruta H, Kurebayashi Y: Tertiary structure-related activity of tick defensin (persulcatusin) in the taiga tick, Ixodes persulcatus. Exp Appl Acarol 2010, 53:71-77.

32. Gong T, Jiang Y, Wang Y, Yang D, Li W, Zhang Q, Feng W, Wang B, Jiang Z, Li M: Recombinant mouse beta-defensin 2 inhibits infection by influenza A virus by blocking its entry. Arch Virol 2010, 155:491-498.

33. Nguyen EK, Nemerow GR, Smith JG: Direct evidence from single-cell analysis that human \{alpha\}-defensins block adenovirus uncoating to neutralize infection. J Virol 2010, 84:4041-4049.

34. Leclercq R: Epidemiological and resistance issues in multidrug-resistant staphylococci and enterococci. Clin Microbiol Infect 2009, 15:224-231.

35. Rice LB: The clinical consequences of antimicrobial resistance. Curr Opin Microbiol 2009, 12:476-481.

36. Heinz FX, Kunz C: Homogeneity of the structural glycoprotein from European isolates of tick-borne encephalitis virus: comparison with other flaviviruses. J Gen Virol 1981, 57:236-274.

37. Pospíšil $L$, Jandásek $L$, Pešek J: Isolation of new strains of tick-borne encephalitis virus, Brno region, summer 1953. (In Czech) Lék listy 1954, 9:3-5.

38. De Madrid AT, Porterfield JS: A simple microculture method for the study of group B arboviruses. Bull World Health Organ 1969, 40:113-121.

39. Kožuch O, Mayer V: Pig kidney epithelial (PS) cells: a perfect tool for the study of flaviviruses and some other arboviruses. Acta Virol 1975, 19:498.

40. Tran D, Tran P, Roberts K, Osapay G, Schaal J, Ouellette A, Selsted ME: Microbicidal properties and cytocidal selectivity of rhesus macaque theta defensins. Antimicrob Agents Chemother 2008, 52:944-953.

41. Cerovský V, Hovorka O, Cvacka J, Voburka Z, Bednárová L, Borovicková L, Slaninová J, Fucík V: Melectin: a novel antimicrobial peptide from the venom of the cleptoparasitic bee Melecta albifrons. Chembiochem 2008, 9:2815-2821.

42. Rahman M, Tsuji N, Boldbaatar D, Battur B, Liao M, Umemiya-Shirafuji R, You M, Tanaka T, Fujisaki K: Structural characterization and cytolytic activity of a potent antimicrobial motif in longicin, a defensin-like peptide in the tick Haemaphysalis longicornis. J Vet Med Sci 2010, 72:149-156.

43. Yang YS, Mitta G, Chavanieu A, Calas B, Sanchez JF, Roch P, Aumelas A: Solution structure and activity of the synthetic four-disulfide bond Mediterranean mussel defensin (MGD-1). Biochemistry 2000, 39:14436-14447

doi:10.1186/1756-3305-4-63

Cite this article as: Chrudimská et al.: Functional characterization of two defensin isoforms of the hard tick Ixodes ricinus. Parasites \& Vectors 2011 4:63. 